So schwankende Anhaltspunkte wie die genannten Wolkenschichten erschweren allerdings die genaue Ableitung der Drehungszeit ungemein. Ebenso schwierig gestaltet sich die Festlegung der Axenlage. Letztere wird allerdings durch das Auftreten von Polarflecken nicht wenig erleichtert. Es gelang dem Verfasser sogar mehrmals (December 1897 und Januar r 898 ) zur Zeit der oberen Conjunction des Planeten, -Aequatorealstreifen ähnlich denen des Planeten Jupiter wahrzunehmen, welche zur Lösung der Doppelaufgabe nicht geringe Dienste zu leisten im Stande wären.

Der Verfasser schloss mit der Hoffnung, dass es dem Spectroskop gelingen möge, schliesslich den verwirtten Knoten der so viel umstrittenen Frage befriedigend und endgültig zu lösen. Die Untersuchungen des Herrn Belopolsky liefern den Beweis, dass wir der Erfüllung dieser Hoffnung um vieles näher gerückt sind.

$$
\text { Rom, } 1900 \text { April } 26 .
$$

Adolf Müller, S. J., Director der Privatsternwarte auf dem Janiculum.

Zusatz des Herausgebers. Ich habe geglaubt, dem Herrn Verfasser einen kurzen Hinweis: auf die beiden von ihn veröffentlichten Schriften nicht versagen zu sollen. Im Uebrigen möchte ich aber doch betonen, dass Herr Belopolsky selbst (vgl. A. N. 364I) seine werthvollen Untersuchungen nur als Versuch die Rotationszeit der Venus zu bestimmen ansieht, ohne den Anspruch zu machen, eine endgültige Lösung der wichtigen Frage herbeigeführt zu haben.

\title{
Vorschlag zu einer Untersuchung an Heliometern.
}

Die in den nächsten Wintermonaten bevorstehende Opposition des Planeten Eros würde bei der grossen Annäherung an die Erde und der hohen Declination für die Sternwarten der nördlichen Halbkugel eine vorzügliche Gelegenheit bieten, die Sonnenparallaxe durch Beobachtung an den Heliometern verschiedener Sternwarten zu bestimmen, wenn dem nicht die geringe Helligkeit entgegenstände, da die Grösse bei Beginn der von Professor Millosevich berechneten Ephemeride im September 1900 nur II.I betrüge und erst gegen Ende derselben im Januar igo I auf 8.4 stiege.

Während also diese Erscheinung von Eros sich zur Parallaxenbestimmung an Heliometern nicht eignet, lässt sich damit eine andere nützliche Untersuchung verbinden, die ich den Beobachtern an Heliometern empfehlen möchte und selbstverständlich auch selbst ausführen werde. Zur Zeit der grössten Helligkeit des Planeten im December rgoo und Januar I90I ist die tägliche Bewegung sehr gross, nämlich $\Delta \alpha \cos \delta=+3$ I.9 und $\Delta \delta=-25 \cdot 7$, also im Bogen grössten Kreises 4 ז!。 im Positionswinkel $129^{\circ}$.

Da die grösste an einem der neueren Heliometer messbare Distanz 2 Grad beträgt, so wird dieser Raum vom Planeten in nicht ganz drei Tagen durchlaufen und wenn es gelänge, den Abstand des Planeten gegen einen im Positionswinkel I 29 Grad belegenen Stern im Verlauf von drei auf einander folgenden Nächten zwischen diesen Grenzen durch häufige Distanzmessungen anzuschliessen, so würde man eine grosse Zahl von Vergleichungen der Messungen unter einander erhalten, sofern man die Bewegung des Planeten als hinreichend bekannt betrachten kann. Sollte letz- teres augenblicklich auch noch nicht der Fall sein, so wird doch durch die obne $Z_{\text {weifel grosse }} Z$ ahl von Beobachtungen, die an zahlreichen Orten während der Wintermonate ausgeführt werden, die Bahn sehr genau bestimmt werden.

Es wird sich daher ein vorzügliches Mittel ergeben, Untersuchungen über die bei Heliometermessungen bemerkten systematischen Fehler anzustellen. Auch für die Untersuchung der fortschreitenden Fehler in den Distanzmessungen an Fadenmikrometern ist die Gelegenheit sehr gtinstig, denn wenn man die Grenze der damit messbaren Distanzen zu etwa ro Minuten annimmt, so wird dieser Raum in einem viertel Tage durchlaufen werden und man könnte durch die Beobachtung in einer Winternacht, um Ermüdung zu vermeiden, mit den nöthigen Ruhepausen, ein grösseres Material zur Untersuchung der Mikrometerschrauben frei von irgend welcher Voraussetzung herbeischaffen.

$\mathrm{Zu}$ erwägen ist dabei noch, ob der Planet genügend lange am Himmel in geeigneter Höhe sichtbar ist. In der Mitte der erwähnten Zeit, also gegen Anfang $190 \mathbf{r}$, ist der Ort des Planeten $2^{\mathrm{h}}$. und $+38^{\circ}$, d. h. für Göttingen circumpolar. Der Planet culminirt um $7^{\mathrm{h}} \cdot 4 \mathrm{M}$. Z. abends in einer Zenithdistanz von 14 Grad und ist daher während eines grossen Theils der Nacht hoch am Himmel und bequem zu beobachten.

Eine andere ganz günstige Gelegenheit zu derartigen Untersuchungen bietet der Planet (6) Hebe, der Juni 27 bei $-6^{\circ}$ Declination um Mitternacht culminirt und im Positionswinkel von etwa 7 r Grad täglich um 16 Bogenminuten fortrückt, also namentlich auf der südlichen Halbkugel während der langen Nächte bequem beobachtet werden könnte.

$$
\text { Göttingen, Mai r } 900 .
$$

Wilhelm Schur.

\section{Beobachtungen auf der Privatsternwarte zu Jena im Jahre 189.9.}

$\begin{array}{rlcc}1899 & \text { Stern } & \text { Phase } & \text { Vergr. } \\ \text { Febr. 18 } & \text { 103 Tauri } & \text { Ed } & 154 \\ 18 & \text { 103 Tauri } & \text { Ah } & 154 \\ \text { März 28 } & \text { 83 Virginis } & \text { Ed } & 154\end{array}$

\section{Sternbedeckungen.}

$$
\begin{aligned}
& \text { M. } z \text {. Jena } \\
& 9^{\mathrm{h}} 54^{\mathrm{m}} \mathrm{I}^{\mathrm{s}} \mathrm{I} \\
& \text { I } 6 \quad 52.3 \\
& \text { I1 } 213.1
\end{aligned}
$$

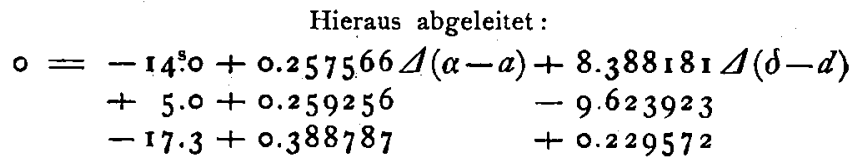

